\title{
Für eine horizontale Arbeitsteilung zwischen nationalem und europäischem Wertpapierhandelsrecht - Gesetzgebung und Methodik
}

Das europäische Kapitalmarktrecht hat in den letzten 50 Jahren das Recht in der europäischen Union umfangreich harmonisiert. Es zielt auf den Schutz der Märkte und der Schaffung eines Binnenmarktes (I.). Die europäische Gesetzgebung schuf aber zu viele Regelungen mit zu wenig Ertrag und erzeugt damit schlechtes Recht mit zu hohen Transaktionskosten (II.). Gefordert wird deshalb eine bessere Arbeitsteilung, die bestimmte, nicht binnenmarktspezifische Bereiche künftig den Mitgliedstaaten überlässt (III.).

\section{Die Regelungsziele des Kapitalmarktrechts}

\section{Rechtsideen des Rechts: Gerechtigkeit, Rechtssicherheit, Sicherstellung von Freiheitsräumen}

Recht zielt auf Gerechtigkeit. ${ }^{1}$ Aristoteles unterscheidet zwischen der ausgleichenden Gerechtigkeit und der verteilenden Gerechtigkeit. ${ }^{2}$ Die ausgleichende Gerechtigkeit (iustitia commutativa) bestimmt, was einem jeden durch das Prinzip der Gegenseitigkeit (Reziprozität) zukommt. Die verteilende Gerechtigkeit (iustitia distributiva) ist regelmäßig nicht in einem Gleichordnungs-, sondern einem Überund Unterordnungsverhältnis angesiedelt. Sie erlaubt korrigierende Eingriffe in das Erziehungs-, Ausbildungs- und Wirtschaftssystem. ${ }^{3}$ So sieht etwa die soziale Marktwirtschaft, wie sie in Deutschland bekannt ist, im Kartellrecht oder zum Schutz des Schwächeren im Miet-, Arbeits- und Verbraucherschutzrecht Korrekturen vor. ${ }^{4}$ Radbruch nimmt diese Gerechtigkeit von Aristoteles auf und nennt

1 Zum Folgenden Möllers Juristische Methodenlehre, 2. Aufl. 2019, § 1 Rn. $106 \mathrm{ff} .=$ S. $38 \mathrm{ff}$.

2 Aristoteles, Nikomachische Ethik, 5. Buch, 4-9. Hierzu Rüthers JZ 2009, 969, 970; Honsell in Staudinger, BGB, Neubearb. 2018, Einl. zum BGB Rn. 113b.

3 R. Dreier JuS 1996, 580, 583.

4 S. Möllers JuS 1999, $1191 \mathrm{ff}$. 
als weitere allgemeine Rechtsideen die Zweckmäßigkeit und den Rechtsfrieden. ${ }^{5}$ Rechtssicherheit und Rechtsfrieden werden dadurch erreicht, dass der Staat verbindliche Regelungen für das Zusammenleben, also Rechtsnormen, setzt. Bürger wissen dann, wozu die einzelnen Rechtsnormen verpflichten oder berechtigen. Der Rechtsfrieden wird aber auch erreicht, indem der Staat jedem Einzelnen Mittel zur Verfügung stellt, um seine Rechte durchzusetzen; in diesem Fall wird der Rechtsfriede durch die gerichtliche Klärung des streitigen Sachverhalts erreicht. Schließlich möchte das Recht Freiheitsräume schaffen. Die Privatautonomie mit ihrer Vertragsfreiheit ist ein wichtiges Prinzip westlicher Gesellschaften. Die Autonomie des Menschen bedingt seine Freiheit. Die Verfassung möchte Freiheitsräume sicherstellen. ${ }^{6}$ Vertragsfreiheit verlangt Selbstbestimmung, so wie Art. 2 Abs. 1 GG die freie Entfaltung der Persönlichkeit formuliert. Coing spricht schließlich von der iustitia protectiva, der begrenzenden Gerechtigkeit. $^{7}$ Einseitiger Machtmissbrauch zulasten anderer muss begrenzt werden. Maßstab ist nicht die Sicherstellung des Gerechten, sondern die Verhinderung des Ungerechten. ${ }^{8}$ Lässt man nun das europäische Kapitalmarktrecht Revue passieren, fragt sich, ob der europäische und der nationale Gesetzgeber diese verschiedenen Varianten des Gerechtigkeitspostulats erreicht haben.

\section{Europäisches Kapitalmarktrecht bis hin zu Lamfalussy und Larosière}

Das europäische Kapitalmarktrecht hat seinen Ursprung in dem Segré-Bericht von $1966^{9}$, die jetzigen europäischen Regelungen stammen vom Aktionsplan Finanzdienstleistungen (Financial Services Action Plan - FSAP) aus dem Jahre $1999^{10}$. Die meisten der heutigen Richtlinien und Verordnungen beruhen auf dem FSAP, etwa die Prospekt-VO Nr. 2017/112911, MiFID II 2014/65/EG, Marktmiss-

5 Radbruch, Grundzüge der Rechtsphilosophie, 1914, S. 82ff.; ders. Rechtsphilosophie, 8. Aufl. 1973, § 9 unterscheidet Gerechtigkeit, Zweckmäßigkeit und Rechtsicherheit; hierzu etwa Kaufmann, Rechtsphilosophie, 2. Aufl. 1997, S. 169 ff.; Larenz, Richtiges Recht, 1979, S. 12 ff., 33 ff. 6 Ähnlich BVerfGE 12, 45, 51 (Kriegsdienstverweigerung I).

7 Coing Grundzüge der Rechtsphilosophie, 5. Aufl. 1993, S. 197.

8 von Hayek, Recht, Gesetzgebung und Freiheit, 1981, S. 62 ff.

9 EWG-Kommission, Der Aufbau eines europäischen Kapitalmarkts: Bericht einer von der EWGKommission eingesetzten Sachverständigengruppe, 1966.

10 Mitteilung der Kommission v. 11.5.1999, Umsetzung des Finanzmarktrahmens: Aktionsplan, KOM (1999) 232 endg.

11 Aus Platzgründen wird auf die genaue Fundstelle europäischer Richtlinien und Verordnungen verzichtet. Alle Rechtsquellen finden sich systematisch unter www.caplaw.de. 
brauchs-VO Nr. 596/2014, Transparenz-RiL 2004/109/EG und Übernahme-RiL 2004/25/EG. Zur Umsetzung der FSAP wurde ein eigenes Rechtssetzungsverfahren von einer Expertenkommission unter Baron von Lamfalussy vorgeschlagen, das Lamfalussy-Verfahren. Drei Ebenen bilden hierbei die Rechtssetzung und eine Ebene die Überwachung. ${ }^{12}$ Die Finanzkrise 2008 führte zu einer Reform der europäischen Aufsichtsstruktur und mehr als 30 Einzelmaßnahmen im Bereich des Banken-, Kapitalmarkt- und Versicherungsrechts, vorgeschlagen durch die Expertengruppe von Larosière ${ }^{13}$. Europäische Aufsichtsbehörden sind nun die ESMA (European Securities and Markets Authority) für den Wertpapiersektor, die EBA (European Banking Authority) für den Bankensektor und die EIOPA (European Insurance and Occupational Pensions Authority) für den Versicherungssektor. Zudem wurde das Lamfalussy-Verfahren novelliert und in diesem Rahmen auch die eingangs genannten Richtlinien und Verordnungen neu verfasst und verschärft. Nach dem reformierten Lamfalussy-Verfahren ${ }^{14}$ erlassen die Kommission, der Rat und das Parlament auf der ersten Ebene Basisrechtsakte, wie Richtlinien oder Verordnungen, Art. 288 Abs. 2, 3 AEUV. Diese werden dann auf der zweiten Stufe durch Durchführungsrechtsakte der Kommission konkretisiert, Art. $290 \mathrm{f}$. AEUV. Daneben kann die jeweils zuständige europäische Aufsichtsbehörde technische Regulierungs- und Durchführungsstandards (RTS und ITS) erlassen, wenn dies in dem Basisrechtsakt vorgesehen ist. Die ESMA-VO Nr. 1095/2010 bestimmt in Art. 8 Abs. 1 lit. a) ausdrücklich, dass die ESMA als Aufsichtsbehörde Leitlinien und Empfehlungen erlassen kann. Als sekundäre Rechtsquellen erzeugen sie mehr als nur faktische Bindungswirkung, weil sie eine Richtigkeitsvermutung begründen und der Mitgliedstaat erklären und darlegen muss, wenn er den Leitlinien nicht nachkommen will. ${ }^{15}$ Um Lücken zu schließen, wurden etwa die OGAW-IV-RiL 2009/65/EG und OGAW-V-RiL 2014/91/EU, die AIFM-RiL 2011/61/ EU, die Leerverkaufs-VO Nr. 236/2012, die EMiR Nr. 648/2012 und die Rating-VO Nr. 1060/2009 erlassen. Zudem wurden die Prospekt-RiL 2003/71/EG und Prospekt-VO Nr. 583/2010, Marktmissbrauchs-VO (MAR) Nr. 596/2014 und Markt-

12 Zum Lamfalussy-Verfahren s. Schlussbericht des Ausschusses der Weisen über die Regulierung der europäischen Wertpapiermärkte v. 15.2.2001, abrufbar unter http://ec.europa.eu/finan ce/securities/docs/lamfalussy/wisemen/final-report-wise-men_de.pdf.

13 De Larosière, The High-Level Group on Financial Supervision in the EU, Report v. 25.2.2009. 14 S. Fn. 13. In der Literatur wird zum Teil von Lamfalussy-II-Verfahren gesprochen, s. etwa Lutter/Bayer/Schmidt Europäisches Unternehmens- und Kapitalmarktrecht, 6. Aufl. 2017, Rn. 14, 45 ff.; Walla BKR 2012, 265, 267; Klöhn in Langenbucher, Europäisches Privat- und Wirtschaftsrecht, 3. Aufl. 2013, § 6 Rn. $21 \mathrm{ff}$.

15 Möllers NZG 2010, 285, 286; ders. Juristische Methodenlehre, 2. Aufl. 2019, § 3 Rn. 73 = S. 103 f.; ihm folgend BVerwG ZIP 2011, 1313 Rn. 26. 
missbrauchs-RiL 2014/57/EU, Transparenz-RiL 2013/50/EU und MiFID II 2014/65/ EU novelliert.

\section{Die Regelungsziele des Kapitalmarktrechts}

\section{a) Schutz der Märkte, Schutz der Anleger, Schutz der Verbraucher}

Bekanntlich dient das Kapitalmarktrecht dem Funktionsschutz der Kapitalmärkte und dem institutionellen sowie individuellen Anlegerschutz. ${ }^{16}$ Die Funktionsfähigkeit des Kapitalmarkts zählt zu den Zielbestimmungen jedes kapitalmarktrechtlichen Gesetzes. Im Kapitalmarktrecht bezieht der Gesetzgeber in seinen Gesetzesvorlagen oder Erwägungsgründen regelmäßig ausdrücklich ökonomische Überlegungen mit ein. ${ }^{17}$ Nur so kann der Markt seine volkswirtschaftliche Funktion erfüllen und das Kapital effizient verteilen. ${ }^{18}$ Zur Allokationseffizienz gehört, dass anlagefähiges Kapital dorthin fließt, wo es am dringendsten benötigt und am erfolgversprechendsten eingesetzt wird. ${ }^{19}$ Wirtschaftswissenschaftlicher Hintergrund der gesetzlichen Informationspflichten ist insoweit die Kapitalmarkteffizienzhypothese. Sie besagt, dass öffentlich zugängliche Informationen sich sofort nach ihrem Bekanntwerden im Marktpreis abbilden. ${ }^{20}$ Damit bezweckt das Kapitalmarktrecht auch den sog. individuellen Anlegerschutz. Man spricht insoweit von zwei Seiten einer Medaille. ${ }^{21}$ Auch in den Erwägungsgründen der einschlägigen Richtlinien finden sich beide Regelungsziele. ${ }^{22}$ Inzwischen ist auch

16 Hopt ZHR 141 (1977), 389, 431.

17 Hierzu Möllers AcP 208 (2008), 1, 5 ff.; Köndgen ZHR Beiheft 74 (2008), 100 ff.; Sester ZGR 2009, $310 \mathrm{ff}$. S. etwa Erwägungsgrund $2 \mathrm{MAR}$.

18 BT-Drucks. 12/6679, S. 48; BT-Drucks. 12/7918, S. 96. Vgl. auch Baums ZHR 167 (2003), 139, 150; Möllers in Möllers/Rotter, Ad-hoc-Publizität, 2003, § 3 Rn. 43; Hopt/Voigt in Hopt/Voigt, Prospektund Kapitalmarktinformationshaftung, 2006, S. 9, 107, 113.

19 S. Erwägungsgrund 1 der Richtlinie 2004/109/EG des Europäischen Parlaments und des Rates zur Harmonisierung der Transparenzanforderungen in Bezug auf Informationen über Emittenten, deren Wertpapiere zum Handel auf einem geregelten Markt zugelassen sind v. 15.12.2004, ABl. EU Nr. L 390, S. 38 (Harmonisierungs-RiL).

20 Zur Informationseffizienz der Kapitalmärkte und der Efficient Capital Market Hypothesis (ECMH), s. etwa Fama 25 J.Fin. 383, 384f. (1970); ders. 46 J.Fin. 1575, 1576 (1991); Fischel 74 Cornell L. Rev. 907 (1989).

21 Hopt ZHR 159 (1995), 135, 159; Grundmann in Großkomm. HGB, Bankvertragsrecht, Zweiter Teil, 5 Aufl. 2017, Rn. 31 = S. 26; Buck-Heeb Kapitalmarktrecht, 10. Aufl. 2018, § 1 Rn. 7 ff. = S. 3 ff. betont darüber hinaus den Funktionenschutz und die Finanzmarktstabilität.

$22 \mathrm{Zu}$ früheren Richtlinien s. Möllers in Möllers/Rotter, Ad-hoc-Publizität, 2003, § 3 Rn. 26. 
der Verbraucherschutz als eigenes Schutzziel hinzugekommen ${ }^{23}$; er erlaubt etwa auch Produktverbote ${ }^{24}$.

\section{b) Level playing field}

Seit der Finanzkrise 2008 haben Gesetzgeber weltweit für Banken, Versicherungen und andere Teilnehmer an den Kapitalmärkten die Regelungen verschärft, etwa in den USA ${ }^{25}$, Großbritannien ${ }^{26}$ oder auch Südafrika ${ }^{27}$. Ziel ist es, bisher noch ungeregelte Märkte zu überwachen. Die Rechtsangleichung diente immer schon der Herstellung des Binnenmarktes. Auch in den letzten Jahren versuchte man mit einer intensiven Regelungsdichte, ein „race to the bottom“ bei den privaten Marktteilnehmern, aber auch bei den Aufsichtsbehörden zu vermeiden. Erklärtes Ziel ist es, ein gemeinsames Recht („level playing field“) herzustellen ${ }^{28}$, also Phänomene wie „gold plating“ oder „cherry picking“29 und letztlich Transaktionskosten durch verschiedene Märkte zu reduzieren ${ }^{30}$. Im Folgenden ist kritisch zu fragen, ob diese Ziele erreicht wurden. Drei Probleme sind in aller Kürze anzusprechen, die unzureichende Lückenfüllung, die Normenüberflutung und die unzureichende Rechtsdogmatik und juristische Methode.

23 Art. 1 Abs. 5 UAbs. 1 S. 2 lit. f) ESMA-VO Nr. 1095/2010; Erwägungsgrund Nr. 2 der LeerverkaufsVO Nr. 236/212.

24 Möllers/Poppele ZGR 2013, 437, 455 ff.; Schäfer FS Marsch-Barner, 2018, S. 471 ff.

25 Dodd-Frank Wall Street Reform and Consumer Protection Act, Pub. L. No. 111-203 v. 21.7. 2010, 124 Stat. 1376 (2010).

26 Financial Services Act 2012 v. 19.12.2012, 2012 c. 21.

27 Implementing a twin peaks model of financial regulation in South Africa v. 1.2.2013, abrufbar unter www.treasury.gov.za/twinpeaks/20131211\%20-020Item\%203\%20Roadmap.pdf.

28 So bereits Hopt/Voigt, Prospekt- und Kapitalmarktinformationshaftung, 2005, S. $2 \mathrm{ff}$.

29 Berichterstatter Edgardo Maria Iozia in der Stellungnahme des Europäischen Wirtschafts- und Sozialausschusses zu dem „Vorschlag für eine Verordnung des Europäischen Parlaments und des Rates über Basisinformationsblätter für Anlageprodukte“, 14./15.11.2012, KOM (2012) 352 endg. 2012/0169 (COD) (2013/C 11/13) unter 3.4.

30 De Larosière, The High-Level Group on Financial Supervision in the EU, Report v. 25.2.2009 Rn. 101; Fleischer/Schmolke NZG 2010, 1241, 1245 f. 


\section{Europäisches Kapitalmarktrecht: zwischen unzureichender Lückenfüllung und Normenüberflutung}

\section{Der gesetzgeberische Regulierungsoverkill}

\section{a) Allgemeines Preußisches Landrecht und die Suche nach dem Recht}

Früher konnte der Anwender mit einer übersichtlichen Reihe von nationalen kapitalmarktrechtlichen Gesetzen und Verordnungen arbeiten. Hinzu kam der Emittentenleitfaden der BaFin. Durch das Lamfalussy-Verfahren gibt es inzwischen sechs Ebenen. Der Umfang der europäischen Gesetze ist inzwischen auf mehrere Tausend Seiten gestiegen. ${ }^{31}$ Inzwischen ist es schon eine hohe Kunst, das Recht überhaupt zu finden. Der größte deutsche Rechtsverlag bietet z.Z. eine Sammlung, welches die aktuellen Rechtstexte abdruckt, überhaupt nicht mehr an. ${ }^{32}$ Erforderlich ist der Rückgriff auf eine Datenbank. ${ }^{33}$ Die Gesetzgebungsmaschinerie erinnert an den Versuch von Friedrich II., mit dem Allgemeinen PreuBischen Landrecht (ALR) und seinen 17.000 Paragraphen alle Lebensverhältnisse abzudecken. Auch aufgrund der Tatsache, dass nur eine Regierungskommission das Recht ergänzen durfte, scheiterte er mit diesem Versuch. ${ }^{34}$ Das ALR war schon bei seiner Verabschiedung überholt und bekanntlich orientierte sich die Pandektenwissenschaft des 19. Jahrhunderts lieber am römischen Recht.

\section{b) Handwerklich schlechtes Recht}

Ein solcher Rechtszustand wäre noch hinnehmbar, wenn man mit der europäischen Normenflut Fälle lösen könnte. Dem ist leider oft nicht so. Unklare Fälle

31 So das mühevolle Zählen von Seiten durch die Mitarbeiter meines Lehrstuhls.

32 Die Textsammlung „Kapitalmarktrecht“ des Beck-Verlages enthielt bisher mit der Marktmissbrauchs-VO Nr. 596/2014 nur eine einzige Verordnung auf der ersten Stufe. Es fehlten alle europäischen Verordnungen der zweiten Stufe. Besserung ist in Sicht, nachdem die Textsammlung ab 2019 von Kollegen Klöhn betreut wird.

33 S. oben Fn. 11.

34 S. die Kabinettsorder vom 14.4.1780, NCC Bd. VI, Nr. 13, Sp. 1935, 1942; Patent wegen Publikation des neuen allgemeinen Landrechts für die Preußischen Staaten vom 5. 2.1794, in: Schering, Allgemeines Landrecht für die Preußischen Staaten, I. Band, Theil I, Titel 1-11, 3. Ausg. 1876, S. XXVI; vgl. auch §§ 46-50 Einl. Preußisches ALR. 
wurden inzwischen zu Hauf gesammelt ${ }^{35}$ : So waren etwa die Vorgaben einer irreführenden Finanzanalyse früher präziser als heute, weil etwa falsche oder irreführende Informationen eindeutig verboten waren. ${ }^{36}$ Hinzu kommen zahlreiche Übersetzungsfehler.

\section{c) Das Nebeneinander von nationalem und europäischem Recht und die Transaktionskosten}

Preistreibend ist zudem das ständige Vorpreschen des deutschen Gesetzgebers. So hat der deutsche Gesetzgeber im Vorfeld zur MiFiD II Gesetze geschaffen, um das Produktinformationsblatt und die Honorarberatung einzuführen. Ökonomisch ist dies wenig effizient: Es erhöht die Transaktionskosten der Marktteilnehmer, weil sich diese in kurzem, zeitlichen Abstand auf unterschiedliche Parameter einstellen müssen. ${ }^{37}$ Die Finanzbranche gerät zum Versuchskaninchen. Kaum ist ein Gesetz erlassen, so wird es wieder modifiziert. ${ }^{38}$

\section{Die unzureichende Lückenfüllung}

\section{a) Fehlende Abstimmung mit Nachbardisziplinen wie dem Kapitalgesellschaftsrecht}

Wegen dem Prinzip der begrenzten Einzelermächtigung des Art. 5 Abs. 1 EUV verbleiben aber weiterhin große weiße Flecken auf der Rechtslandschaft, die nicht vom europäischen Kapitalmarktrecht erfasst sind. Das europäische Gesellschaftsrecht ist zwar durch zahlreiche Richtlinien und Verordnungen harmonisiert. ${ }^{39}$ Unklar bleiben aber die Bereiche, an deren Stelle sich Gesellschaftsrecht und Kapitalmarktrecht überschneiden. So könne eine Information, etwa der

35 S. etwa Simonis AG 2016, 651ff.

36 Ausgeführt bei Möllers NZG 2018, 649, 651.

37 Kritisch: Möllers/Wenniger, Stellungnahme v. 16.6.2009 zum Regierungsentwurf eines Gesetzes zur Neuregelung der Rechtsverhältnisse bei Schuldverschreibungen aus Gesamtemissionen und zur verbesserten Durchsetzbarkeit von Ansprüchen von Anlegern aus Falschberatung (BTDrucks. 16/12814) v. 29.4.2009, S. $10 \mathrm{ff}$; Jesch/Siemko BB 2014, $2570 \mathrm{ff}$.

38 Möllers ZEuP 2016, 325, $335 \mathrm{ff}$.

39 Für einen Überblick s. Habersack/Verse Europäisches Gesellschaftsrecht, 5. Aufl. 2019; Grundmann Europäisches Gesellschaftsrecht, 2. Aufl. 2011; Möllers in Schulze/Zuleeg/Kadelbach, Europarecht, 4. Aufl. 2020, § 18 (im Erescheinen); Teichmann in Gebauer/Teichmann, Europäisches Privat- und Unternehmensrecht, 2016, § 6. 
vorzeitige Rücktritt des Daimler-Vorstandsvorsitzenden Schrempp, schon dann eine Ad-hoc-pflichtige Information sein, wenn der Entschluss soweit „,verdichtet“ war, dass er nicht mehr umkehrbar war. Auf die Zustimmung des Aufsichtsrates käme es dann nicht mehr an. ${ }^{40}$ Oder: bis dato war heftig umstritten, ob der kapitalmarktrechtliche Anlegerschutz oder der gesellschaftsrechtliche Kapitalerhaltungsgrundsatz vorgeht. Der EuGH entschied, dass eine Schadenersatzklage eines Investors zulässig ist und nicht gegen den gesellschaftsrechtlichen Grundsatz der Kapitalerhaltung verstößt. ${ }^{41}$ Vom EuGH noch nicht entschieden ist die umstrittene Frage, ob die deutsche Wissenszurechnung im Konzern Auswirkungen auf die Ad-hoc-Publizität hat. ${ }^{42}$

\section{b) Fehlende intradisziplinäre Abstimmung zwischen öffentlichem Recht, Zivil-, Straf- und Prozessrecht}

An anderer Stelle wurde schon ausgeführt, dass das europäische Kapitalmarktrecht hauptsächlich mit den Mitteln des öffentlich-rechtlichen Aufsichtsrechts durchgesetzt wird. ${ }^{43}$ Zivilrechtliche Anspruchsgrundlagen sind rar; unklar bleibt, in welchem Umfang das Kapitalmarktrecht deliktsrechtliche Schutzgesetze darstellen und damit das nationale Zivilrecht nutzbar gemacht werden kann. ${ }^{44} \mathrm{We}$ nig effektiv ist auch die Verbindung zum Strafrecht, wenn die regionale Staatsanwaltschaft nicht über das erforderliche Know-how verfügt. ${ }^{45}$ Auch was die Verbindung der verschiedenen Rechtswege betrifft, ist das Kartellrecht deutlich weiter. ${ }^{46}$ Auf europäischer Ebene fehlen in weitem Umfang zivilrechtliche Scha-

40 EuGH v. 28.6.2012, Rs. C-19/11, ECLI:EU:C:2012:397 Rn. 49 (Geltl); Möllers/Seidenschwann NJW 2012, 2762, 2764.

41 EuGH v. 19.12. 2013, Rs. C-174/12, ECLI:EU:C:2013:856, Rn. 39f. (Alfred Hirmann/Immofinanz AG); so bereits Möllers BB 2005, 1637, 1641; ders. FS Baums, 2017, S. 805, 824.

42 Zum Streitstand etwa Klöhn in Klöhn, Marktmissbrauchsverordnung, 2018, Art. 17 Rn. $111 \mathrm{ff}$.

43 Möllers NZG 2018, 649, 653; ders. ZEuP 2016, 325, $345 \mathrm{ff}$.

44 Zur MiFiD II s. Möllers ZEuP 2016, 325, 350. Umstritten auch für Artt. 12, 15, 17 MAR. Für die Schutzgesetzfähigkeit etwa Hellgardt AG 2012, 154, 165; Seibt ZGR 177 (2013), 388, 424f.; dagegen Buck-Heeb Kapitalmarktrecht, 10. Aufl. 2019, § 6 Rn. 454 ff., 551 = S. 139 ff., 170; Markworth ZHR 183 (2019), 46, 64 ff. Offen, weil letztlich der EuGH entscheidet, Möllers in Derleder/Knops/Bamberger, Deutsches- und Europäisches Kapitalmarktrecht, 3. Aufl. 2017, § 84 Rn. 39.

45 Kritisch Möllers NZG 2018, 649, 653.

$46 \mathrm{Zu}$ diesen Forderungen bereits Möllers/Pregler ZHR 176 (2012), 144, 162; zustimmend Maume ZHR 180 (2016), 358, 365 ff. S. jetzt die Schadensersatz-RiL 2014/104/EU. Hierzu Kersting VersR 2017, $581 \mathrm{ff}$; Weitbrecht NJW 2017, $1754 \mathrm{ff}$. 
denersatzansprüche; zudem fehlt die Möglichkeit von Gruppenklagen ${ }^{47}$. Letztlich ist auch der Missstand zu nennen, dass zurzeit ein europäisches Fachgericht im europäischen Gesellschafts- und Kapitalmarktrecht fehlt. Der EuGH ist schon aus Kapazitätsgründen nicht in der Lage, die erforderliche Rechtsprechungsdichte zu schaffen, die ein „living law“ verlangen würde.

\section{Komplexität juristischer Methoden}

\section{a) Die komplexe juristische Methodik bei der Arbeit mit europäischen Richtlinien und Verordnungen}

Der Richter und damit jeder Rechtsanwender muss europäisches Recht autonom, d.h. unabhängig von seinem nationalen Normverständnis auslegen und diesem zur Durchsetzung verhelfen. Die autonome Auslegung kann dazu führen, dass sie nationalem Recht widerspricht, aber als höherrangiges Recht dem nationalen Recht vorgeht. Gegebenenfalls hat der Rechtsanwender rechtsvergleichend $\mathrm{zu}$ arbeiten. Zudem ist das nationale Recht unionsrechtskonform und richtlinienkonform auszulegen. Verstößt ein Gericht gegen Unionsrecht, ist eine Staatshaftung für judikatives Unrecht aufgrund des unionsrechtlichen Staatshaftungsanspruchs denkbar. ${ }^{48}$ Die Arbeit mit Richtlinien ist komplex, weil der Rechtsanwender fünf Prüfungsschritte einzuhalten hat; zudem kann er nicht vorhersagen, wie weit etwa eine richtlinienkonforme Rechtsfortbildung zulässig ist oder ob im überschießenden Bereich ${ }^{49}$ der Norminhalt der Richtlinie zur Anwendung kommt. Aber auch die Arbeit mit Verordnungen ist nicht einfacher. Schon nach dem Wortlaut sind 24 verschiedene Sprachfassungen zu beachten. Europäische Richtlinien sind in nationales Recht umzusetzen, so dass der Rechtsanwender wie bisher mit dem WpHG als deutscher Rechtsquelle zu arbeiten hat. Die europäische Verordnung verlangt dagegen die unmittelbare Arbeit mit dem europäischen Normtext und damit eine autonome Auslegung, die unabhängig vom nationalen Vorverständnis arbeitet. Fehlerhaft ist damit der unmittelbare Rückgriff auf das nationale Vorverständnis, also unverzüglich auto-

47 Die EU hat einen neuen Entwurf einer Verbandklage (v. 11.4. 2018, EU (COM (2018) 184 final) veröffentlicht. S. hierzu Gsell/Möllers (eds.) Enforcing Consumer and Capital Market Law in Europe, 2020 (im Erscheinen).

48 EuGH v. 30.9.2003, Rs. C-224/01, EU:C:2003:513, Rn. 122f. (Köbler).

$49 \mathrm{Zu}$ den einzelnen Fragen s. Möllers Juristische Methodenlehre, 2. Aufl. 2019, § 12 Rn. 63 ff., 90, 102 = S. 429, 436, 439. 
matisch mit der Legaldefinition des $§ 121$ BGB gleichzusetzen. ${ }^{50}$ Eine Arbeit mit der Gesetzgebungsgeschichte oder der Systematik ist nur bedingt möglich. ${ }^{51}$ Wenn nach dem Sinn eines europäischen Begriffs gefragt wird, wäre rechtsvergleichendes Arbeiten vonnöten, wozu der Rechtsanwender bei noch 28 Mitgliedstaaten aber nicht in der Lage ist. ${ }^{52}$

\section{b) Der Verlust an rechtsdogmatischer Sicherheit}

Akzeptiert man die Prämisse, dass der Gesetzgeber, aber auch eine Aufsichtsbehörde, nicht alle Lebensbereiche lösen kann, dann bleibt Recht lückenhaft. Gerichte sind nicht nur zur Rechtskontrolle von Exekutive und Legislative aufgerufen, sondern auch zur Lückenfüllung. Dazu greifen sie nicht nur auf die Gesetze, sondern auch auf die Rechtsdogmatik zurück. Rechtsdogmatik konkretisiert Gesetzestext und Rechtsprechung. Sie bildet eine Tiefenstruktur der Rechtsordnung aus einer Vielzahl miteinander verbundener Ordnungsbegriffe, Lehrsätze, Typologien, Figuren und Leit- und Strukturgedanken. Man kann sie mit der Grammatik vergleichen, weil die Sprache - hier die Rechtsnormen - ohne Kenntnis der Grammatik nicht verstanden und angewendet werden können. ${ }^{53}$ Durch die Ordnung und Systematisierung des vorhandenen Rechtsstoffs schafft die Rechtsdogmatik erst die Möglichkeit zur Differenzierung, die dann von der Rechtsprechung genutzt werden kann, um neue und zugleich systemgerechte Lösungen für bislang nicht gelöste Probleme zu finden. ${ }^{54}$ Die Rechtsdogmatik dient der Gerechtigkeit, weil sie das Problem in Beziehung zu bisherigen Entscheidungen setzt und mit diesem erweiterten Blickwinkel eine gerechte Lösung sucht. Gleichheit, Ungleichheit und Ähnlichkeit sowie deren jeweils entsprechende Behandlung werden dadurch transparent. ${ }^{55}$ Die verschiedenen Zwischenschritte des Rechts, welche die Rechtsdogmatik erzeugt, führen zu einer Vermutungswirkung. Wenn der Rechtsanwender im konkreten Fall auf diese Zwischenschritte oder Prinzi-

50 So aber Buck-Heeb Kapitalmarktrecht, 10. Aufl. 2019, § 6 Rn. 486 = S. 150; Klöhn in Marktmissbrauchsverordnung, 2018, Art. 18 Rn. 105. Richtigstellend vorher schon Möllers FS Horn, 2006, S. 473 ff.; Sethe in Assmann/Schneider/Sethe, WpHG, 6. Aufl. 2012 §§ 37b, c Rn. 105.

51 Kritisch Klöhn in Klöhn, Marktmissbrauchsverordnung, 2018, Einl. Rn. 65 ff., 89 ff.

52 Möllers ZEuP 2016, 325, 352f. Auch die neuen Kommentare zur Marktmissbrauchsverordnung leisten diese rechtsvergleichende Arbeit (noch) nicht.

53 Herberger, Dogmatik, 1981, S. 37 f., 74 ff., 119, 257 ff. mit umfangreichen Nachweisen zum römischen Recht; Jansen ZEuP 2005, 750, 754.

54 Rüthers/Fischer/Birk Rechtstheorie, 10. Aufl. 2018, Rn. 326.

55 Stürner JZ 2012, 10, 11. 
pien zurückgreifen kann, muss er diese nicht mehr ausführlich begründen, weil sie vorab schon durch Rechtsprechung und Rechtsliteratur entwickelt worden waren. ${ }^{56}$ So wurde etwa der Schadensbegriff der $§ 97 \mathrm{f}$. WpHG durch Rechtsliteratur und Rechtsprechung in den letzten zwei Jahrzehnten entwickelt. ${ }^{57}$ Die Rechtsdogmatik verlangt aber eine praktikable juristische Methode, verlässliche Rechtsquellen und eine darauf aufbauende Rechtsliteratur und Rechtsprechung. Die Schnelllebigkeit und Komplexität des europäischen Kapitalmarktrechts stehen dem entgegen. Es ist deshalb wenig erstaunlich, dass die Rechtsdogmatik im Kapitalmarktrecht, die Arbeit mit Prinzipien, eher unterentwickelt ist. ${ }^{58}$ Die deutsche Rechtsprechung kann hingegen teilweise, expressis verbis im Bereich der Anlageberatung, auf das dogmatische System des nationalen Rechts aufbauen..$^{59}$

\section{Untaugliches Recht: Das Verfehlen der Regelungsziele und der Gerechtigkeitsparameter}

Die Bilanz ist aus dreierlei Gründen ernüchternd. Letztlich werden die oben genannten Regelungsziele nicht erreicht. Der Institutionenschutz wird nicht erreicht, wenn durch Short-Sell-Attacken der Eindruck entsteht, an deutschen Börsen dürfte organisierte Kriminalität nach Wildwest-Manier walten und schalten. ${ }^{60}$ Dem individuellen Anlegerschutz wird nicht gedient, wenn die Kosten für die Anlageberatung so in die Höhe getrieben werden, dass sich die Banken von der Anlageberatung zurückziehen. ${ }^{61}$ Des Weiteren ist dem Verbraucher nicht gehol-

56 Esser, Vorverständnis und Methodenwahl in der Rechtsfindung, 1970, S. 88 f.; Brohm VVDStRL 30 (1972), 245, 247; Vesting Rechtstheorie, 2. Aufl. 2015, Rn. 21.

57 Hopt WM 2013, 101, $106 \mathrm{f}$.

58 Ein positives Gegenbeispiel bilden dagegen die Prinzipien von Art. 3 der Übernahme-RiL 2004/25/EG, umgesetzt in § 3 WpÜG. S. auch Grundmann in Großkomm. HGB, Bankvertragsrecht, Zweiter Teil, 5. Aufl. 2017, Rn. 8 ff., 251 ff. = S. 12 ff., 294 ff.

59 So hat die Rechtsprechung den Grundsatz der rationalen Anlegerentscheidung in Auseinandersetzung mit der Rechtsliteratur entwickelt, der dem geschädigten Anleger die Rückerstattung des gezahlten Kaufpreises gewährt, s. BGHZ 192, 90, 109 ff. (IKB); BGHZ 160, 149, 153 f. (Infomatec II); Assmann ZBB 1989, 49, 57 ff.; Möllers ZGR 1997, 334, 338; ders. FS Baums, 2017, S. 805, 817 ff.; Fleischer 64. DJT, 2002, F 27.

60 Möllers NZG 2014, 361, 368; ders. NZG 2018, 649, 658; Maume ZHR 180 (2016), 358, 362.

61 S. die empirische Umfrage von Mansen, Die neuen Anlageberatungsregelungen der MiFID II, 2018, S. 375 ff. sowie die Stellungnahmen des BVI, des ppi, des Genossenschaftsverbandes, der Deutschen Bank, der IHK zur MiFiD II, abrufbar unter www.caplaw.de. 
fen, wenn er in Informationen erstickt. ${ }^{62}$ Dem Anleger werden so Steine statt Brot gegeben. Und effizient sind hohe Transaktionskosten ebenfalls nicht. Das Level playing field wird nicht erreicht, wenn letztlich der Rechtsanwender auf die Suche nach dem europäischen Recht verzichtet. Sehnsüchtig wird in Deutschland die Überarbeitung des Emittentenleitfaden herbeigesehnt. In Großbritannien gilt selbiges für das FSA Handbook. Ein Auseinanderlaufen der vermeintlichen Rechtspraxis in den verschiedenen Mitgliedstaaten ist auch zu konstatieren, wenn die Aufsichtsbehörden in den verschiedenen Mitgliedstaaten das Recht weiterhin unterschiedlich handhaben. Der Begriff der unverzüglichen Ad-hoc-Mitteilung wird in Deutschland viel strenger gehandhabt als in anderen Mitgliedstaaten. ${ }^{63}$ Eine vergleichbare Rechtspraxis, ein „level playing field“, wird so nicht erzeugt. Neben den konkreten Regelungszielen werden aber auch die eingangs genannten Gerechtigkeitsparameter nicht erfüllt: Rechtssicherheit und Rechtsfriede sind tangiert, wenn Recht nicht überwacht wird und der konkrete Schaden rechtlich nicht geltend gemacht werden kann. Produktverbote, aber auch überbordende Transaktionskosten schränken Freiheitsräume unangemessen ein. In dieser Form ist das europäische Kapitalmarktrecht unbefriedigend, ja untauglich.

\section{Eine bessere Arbeitsteilung zwischen EU und den Mitgliedstaaten als Modell der Zukunft}

Das jetzige Kapitalmarktrecht ist mit seiner Normenflut und den gleichzeitig vorhandenen weiteren Lücken untauglich. Eine vertikale Arbeitsteilung, welche die öffentlich-rechtliche Rechtsdurchsetzung auf europäischer Ebene regelt und die zivilrechtrechtliche Rechtsdurchsetzung den Mitgliedstaaten überlässt, erscheint wenig überzeugend (a). Stattdessen sollte stärker auf eine horizontale Trennung geachtet werden, so dass die EU nur für binnenmarktrelevante Gebiete zuständig ist, während ohne einen solchen Bezug die Zuständigkeit bei den Mitgliedstaaten verbliebe (b). Damit könnten zahlreiche Prinzipien und Ziele des europäischen Rechts umgesetzt werden (c).

62 S. hierzu die Studie von Paul/Schröder/Schumacher, Auswirkungsstudie MiFiD II/MiFIR und PRIIPs-VO: Effektivität und Effizienz der Neuregelungen vor dem Hintergrund des Anleger- und Verbraucherschutzes, Feb. 2019.

63 Während früher ein Wochenendbereitschaftsdienst für eine unvorhergesehene Ad-hoc-Mitteilung nicht gefordert wurde, verlangt die BaFin inzwischen eine möglichst schnelle endgültige Entscheidung. A.A. Veil/Brüggemeier in Meier/Veil/Rönnau, Handbuch zum Marktmissbrauchsrecht, 2018, Rn. 114 = S. 256 nach denen sich die Verwaltungspraxis nicht geändert hätte. 


\section{Der status quo: die wenig überzeugende vertikale Arbeitsteilung zwischen EU und den Mitgliedstaaten}

Das europäische Kapitalmarktrecht existiert zwar seit 50 Jahren, bleibt aber wegen der fehlenden Abstimmung mit dem Gesellschaftsrecht und der mangelnden Korrelation von öffentlichem Recht und Zivilrecht immer noch ein Torso. Auch auf nationaler Ebene ist die Rechtslage höchst unterschiedlich. ${ }^{64}$ In Deutschland gibt es einige spezialgesetzliche Haftungsgrundlagen, etwa für fehlerhafte Prospekte $^{65}$, Ad-hoc-Mitteilungen ${ }^{66}$ oder auch fehlerhafte Angebotsunterlagen ${ }^{67}$. Zwar existiert eine umfangreiche zivilrechtliche Rechtsprechung zur fehlerhaften Anlageberatung. ${ }^{68}$ Diese Rechtsprechung führt aber zu kuriosen Ergebnissen: Zwar laufen die öffentlich-rechtlichen und zivilrechtlichen Pflichten teilweise parallel. ${ }^{69}$ Der nationale zivilrechtliche Pflichtenstandard kann aber auch niedriger sein, etwa wenn es der BGH ablehnt, den Normen des öffentlichen Rechts einen Schutzgesetzcharakter für den Geschädigten im Sinne von § 823 Abs. 2 BGB zuzubilligen und damit einen zivilrechtlichen Schadensersatzanspruch zu gewähren. ${ }^{70}$ Im Gegensatz zur deutschen Rechtslage hat etwa der österreichische Gerichtshof einzelnen Wohlverhaltensregeln zivilrechtliche Wirkung zugesprochen. ${ }^{71}$ Vergleichbares gilt für Italien durch den Kassationshof. ${ }^{72}$ In Schweden

64 S. ausführlich die Studie von Hopt/Voigt, Prospekt- und Kapitalmarktinformationshaftung, 2006.

$65 \S \S 21,22$ WpPG; s. etwa BGH, NJW 1982, 2823 (Beton- und Monierbau); BGHZ 195, 1 (Wohnungsbaugesellschaft Leipzig West); BGH, NZG 2015, 20 (Telekom); hierzu Möllers/Steinberger NZG 2015, $329 \mathrm{ff}$.

$66 \mathrm{Zu}$ den einschlägigen Haftungsgrundlagen und Urteilen, s. ausführlich Möllers/Leisch in Hirte/Möllers, Kölner Kommentar zum WpHG, 2. Aufl. 2014, §§ 37b, c Rn. 1 ff.

67 Möllers in Hirte/Möllers, Kölner Kommentar zum WpHG, 2. Aufl. 2014, § 12 Rn. 1 ff.; Rechtsprechung zu § 12 WpÜG ist allerdings noch nicht ergangen.

68 Diese ist inzwischen uferlos, Nachweise bei Edelmann in Assmann/Schütze, Handbuch des Kapitalanlagerechts, 4. Aufl. 2015, §§ $3 \mathrm{f}$.

69 Das gilt etwa für das „know-your customer“ und „know your product“-Prinzip, das sich einerseits auf den Art. 11 Abs. 1 Spiegelstrich 4, 5 der Wertpapierdienstleistungs-RiL 93/22/EWG ergab und andererseits vom BGH entwickelt wurde, BGHZ 123, 126, 128 ff. (Bond); s. Möllers in Hirte/Möllers, Kölner Kommentar zum WpHG, 2. Aufl. 2014, § 31 Rn. 338.

70 BGHZ 186, 58 Rn. 26 ff. zu § 34a Abs. 1 S. 1 WpHG; BGHZ 175, 276 Rn. 18 m.w.Nachw. zu § 32 Abs. 1 Nr. 1 WpHG; BGHZ 192, 90, 98 ff. (IKB) zu § 20a WpHG.

71 OGH v. 20.4.2005, Az. 7 Ob 64/04v, ÖBA 2005, 721, 725; OGH v. 20.1.2005, Az. 2 OB 236/04a, ÖBA 2009, 635, 640; auch zur Marktpreismanipulation nach §48a Abs. 1 BörseG, s. OGH v. 24.1. 2013, Az. 8 Ob 104/12w, ÖBA 2913, 438/1922 unter 6.2. m.w.Nachw.; OGH v. 15.3.2012, Az. 6 Ob 28/ 12d, RIS-Justiz RS0127724 unter 3.4.; abrufbar unter www.ris.bka.gv.at. 
existiert ein eigenes Gesetz, welches Anlegern Schadensersatzansprüche bei fehlerhafter Anlageberatung einräumt. ${ }^{73}$ Schließlich bleibt noch ein letzter Missstand: In Deutschland existieren divergierende Entscheidungen verschiedener Rechtswege, mit dem Resultat, dass sich öffentliches Recht und Zivilrecht widersprechen können. ${ }^{74}$ Hinzu kommen Unterschiede bei der Rechtsdurchsetzung. In Deutschland gibt es etwa das Kapitalanlegermusterverfahrensgesetz (KapMuG), das nach der Novellierung auch gebündelte Schadensersatzklagen bei fehlerhafter Beratung zulässt. ${ }^{75}$ Eine Besserung ist auch hier nicht in Sicht, da eine Einigung auf europäischer Ebene über zivilrechtliche Ansprüche noch aussichtslos erscheint. Das oben gewünschte „level playing field“ wird damit ein weiteres Mal verfehlt. Nicht nur die unterschiedliche Rechtsanwendung, sondern auch die unterschiedliche Rechtsdurchsetzung beeinträchtigt den Binnenmarkt.

\section{Für eine horizontale Arbeitsteilung zwischen EU und den Mitgliedstaaten de lege ferenda}

De lege ferenda könnte man überlegen, die Zuständigkeit im Rahmen des Kapitalmarktrechts horizontal zu trennen: Nur für binnenmarktrelevante Bereiche wäre die Europäische Union zuständig. Vorbilder gibt es zahlreiche. Bereits bei den Grundfreiheiten hält die Rechtsprechung nach der Keck-Rechtsprechung nur noch Handlungen für relevant, die den Binnenmarkt beeinflussen. ${ }^{76}$ Auch im europäischen Kartellrecht gibt es die Unterscheidung zwischen dem nationalen und dem europäischen Markt. Entscheidend ist, ob die Wettbewerbsbeschränkung geeignet ist, den Handel zwischen den Mitgliedstaaten zu beeinträchtigen,

72 Cass. Civ. v. 17.2.2009, n. 3773, Danno e Responsabilità 2009, 503; s. auch Perrone/Valente 13 EBOR 31, 33 (2012).

73 Walla 22 EBLR 211, 218f. (2011).

74 Die BaFin verurteilte die Daimler AG zu einem Bußgeld wegen verspäteter Ad-hoc-Mitteilung, das OLG Stuttgart sprach die Daimler AG dagegen frei; s. OLG Frankfurt NJW 2009, 1520 einerseits und OLG Stuttgart NZG 2007, 352 andererseits; hierzu Möllers NZG 2008, 330 ff.; Möllers/Seidenschwann NJW 2012, 2762, 2764; Wundenberg ZGR 2015, 124, 155.

75 Gesetz über Musterverfahren in kapitalmarktrechtlichen Streitigkeiten (Kapitalanleger-Musterverfahrensgesetz - KapMuG) v. 19.10.2012, BGBl. I, S. 2182; hierzu etwa Hess/Reuschle/Rimmelspacher Kölner Kommentar zum KapMuG, 2. Aufl. 2014; Möllers/Leisch in Hirte/Möllers, Kölner Kommentar zum WpHG, 2. Aufl. 2014, §§ 37b, 37c Rn. 523ff. m.w.Nachw.

76 Grundlegend EuGH v. 24.11.1993, Rs. C-267/91 u.a., EU:C:1993:905 Rn. 16 (Keck und Mithouard) und die Unterscheidung von produktbezogenen und vertriebsbezogenen Maßnahmen. 
Art. 101 Abs. 1, 102 Abs. 1 AEUV. ${ }^{77}$ Dem liegt die richtige Überlegung zugrunde, dass die EU primär für Bereiche zuständig sein soll, die den Binnenmarkt beeinflussen. Damit würde einerseits die EU noch stärker die Kompetenzen erhalten, die in hohem Maße für den Binnenmarkt relevant sind. Und andererseits wären die Mitgliedstaaten für die Bereiche ausschließlich zuständig, die sich nicht auf den europäischen Binnenmarkt auswirken.

\section{a) Eine effektive europäische Aufsicht für binnenmarktrelevante Bereiche}

\section{aa) Der Vergleich mit der SEC}

Die Security Exchange Commission (SEC), die US-amerikanische Wertpapieraufsicht, existiert seit nunmehr fast 80 Jahren und ist der weltweite Maßstab für effektives Handeln. ${ }^{78}$ Sie geht wirkungsvoll gegen Marktmanipulationen vor. Was effektive Aufsicht bedeutet, verdeutlicht auch der VW-Dieselskandal in den USA. Das US-amerikanische Justizministerium verordnete den Unternehmen Bilfinger, Daimler, Siemens und Volkswagen einen Monitor, der die Unternehmen überwacht und an das US-Justizministerium berichtet. Dies ist ein Beispiel für die effektive Durchsetzung von Recht. ${ }^{79}$ In der EU kann vielleicht nur die Generaldirektion Wettbewerb von ihrer Durchschlagskraft mit der SEC verglichen werden. Sie erwies sich in den letzten 60 Jahren auch gegen ausländische Unternehmen als sehr wirkungsvoll, die auf dem europäischen Markt rechtswidrig handelten. Berühmte Verfahren wurden gegen Microsoft, Google oder jüngst Qualcomm ${ }^{80}$ angestrengt.

\section{bb) Bereits vorgenommene Übertragungen von Zuständigkeiten an die ESMA}

Die Kompetenz einer europäischen Aufsicht im Bereich des Kapitalmarktrechts sollte - ähnlich wie im Wettbewerbsrecht - immer für grenzüberschreitende Fälle bestehen, weil hier der Binnenmarkt berührt ist. Immerhin hat die Restrukturie-

77 Hierzu etwa Eilmansberger/Krius in Streinz, EUV/AEUV 3. Aufl. 2018, Art. 101 AEUV Rn. 26 ff.; Weiß in Callies/Ruffert, EUV/AUEV, 5. Aufl. 2016, Art. 101 AEUV Rn. $124 \mathrm{ff}$.

78 Hazen The Law of Securities Regulation, $7^{\text {nd }}$ ed. 2017, Chap. $16=$ S. 675 ff. Für die Marktmanipulation s. etwa Möllers NZG 2018, 649, 654.

79 S. Zwiebel/Lohmeier Compliance-Berater 7/2016, 250 ff.; Schneider Compliance-Berater 12/ 2017, 441 ff.; Freytag FAZ v. 10.2.2018, S. 26.

80 Europäische Kommission, Pressemitteilung v. 24.1.2018, Kartellrecht: Kommission verhängt Geldbuße von 997 Mio. EUR gegen Qualcomm wegen Missbrauchs einer marktbeherrschenden Stellung, abrufbar unter http://europa.eu/rapid/press-release_IP-18-421_de.htm. 
rung des europäischen Kapitalmarktrechts auch eine Aufwertung europäischer Behörden mit sich gebracht. In der Vergangenheit waren ausschließlich die Mitgliedstaaten für das Kapitalmarktrecht zuständig. Dies wurde durch das reformierte Lamfalussy-Verfahren geändert. Zu Recht wurde die Kompetenz für unmittelbar binnenmarktrelevante Bereiche der ESMA übertragen. Die ESMA hat inzwischen die Zuständigkeit gegenüber Ratingagenturen, ${ }^{81}$ ausnahmsweise aber auch im Bereich von Leerverkäufen ${ }^{82}$ und Benchmarks ${ }^{83}$.

\section{cc) Shortsell-Attacken und irreführende Finanzanalysen}

Die ESMA oder vielleicht sogar die Generaldirektion (GD) Finanzstabilität, Finanzdienstleistungen und Kapitalmarktunion ${ }^{84}$ könnten künftig für weitere Bereiche zuständig sein, welche systemrelevant sind, weil sie die Märkte massiv beeinflussen können. In jüngster Zeit sehen sich an deutschen Börsen zahlreiche börsennotierte Unternehmen Angriffen von Marktteilnehmern ausgesetzt, die immer eine ähnliche Strategie umsetzen: Short Seller spekulieren mit Leerverkäufen auf fallende Kurse und verbreiten vorher über Twitter oder ähnliche Medien negative Gerüchte oder negative Finanzanalysen ${ }^{85}$. In Deutschland waren etwa die Unternehmen Aurelius, Ströer, Wirecard oder ProSiebenSat.1 Media von Leerverkaufsattacken betroffen. ${ }^{86}$ Die Kurse stürzten innerhalb kürzester Zeit um

81 Art. 5b der konsolidierten Fassung der Rating-Verordnung (EU) Nr. 1060/2009. Kritisch zum früheren Rechtszustand Möllers JZ 2009, 861.

82 Art. 28 Leerverkaufs-Verordnung Nr. 236/2012.

83 Art. 37 ff. Benchmark-Verordnung Nr. 2016/1011. Hierzu umfassend Brosig, Benchmark-Manipulation, Eine ökonomische und regulatorische Analyse des LIBOR Manipulationsskandals, 2018. $84 \mathrm{Zu}$ ihren Zuständigkeiten s. https://ec.europa.eu/info/departments/financial-stability-finan cial-services-and-capital-markets-union_de\#responsibilities.

85 Das WpHG verwendete in seiner Fassung bis zum 2.7.2016 den Begriff „Finanzanalyse“ (§ 34 b WpHG a. F.). Seit dem ersten Finanzmarktnovellierungsgesetz (1. FiMaNoG) wurde der Begriff an die europäischen Rechtsakte angepasst, sodass nun von „Anlageempfehlung“ gesprochen wird (§ 85 WpHG). Ausführlich zum Folgenden Möllers NZG 2018, 649ff.

86 Zum Sachverhalt etwa Schockenhoff/Culmann AG 2016, 517, 518. Zu ProSiebenSat.1, s. http:// www.deraktionaer.de/aktie/prosiebensat-1-362400.htm. Die jüngste Attacke ging im Frühjahr 2019 gegen Wirecard und trieb den Kurs von 170 auf unter $100 \mathrm{EuR}$, s. Wirecard-Aktienkurs fällt um 20 Prozent, FAZ.NET v. 7.2. 2019, https://www.faz.net/aktuell/wirtschaft/unternehmen/naech ster-medienbericht-wirecard-aktienkurs-faellt-um-20-prozent-16029424.html\#void; Wirecard-Aktie: Das ist wirklich außergewöhnlich, DER AKTIONÄR v. 15.2.2019, http://www.deraktionaer.de/ aktie/wirecard-aktie-fakten-wissen-leerverkaeufer-deutsche-bank-deutsche-boerse-verlust-topverlierer-hdax-boersenbriefing-442690.htm. 
bis zu 30\% ab. ${ }^{87}$ Die Short Seller strichen ihren Gewinn ein, indem sie die verkauften Aktien nach dem Kurssturz günstig zurückerwerben konnten. In den USA existiert inzwischen eine Finanzindustrie aus Analysten, Hedgefonds und Kanzleien, die mit Leerverkaufsattacken versucht, die Kurse von börsennotierten Unternehmen zum Fallen zu bringen. ${ }^{88}$ Jährlich gibt es weltweit deutlich mehr als 100 solcher Leerverkaufsattacken. ${ }^{89}$

Selbst wenn man in Deutschland mit einer Eingreiftruppe und einer zentralen Schwerpunktstaatsanwaltschaft effektiver werden würde, wäre dies nur ein erster wichtiger Schritt. Bei noch 28 Mitgliedstaaten hätten die Short Seller weiterhin leichtes Spiel, auf Börsen anderer Mitgliedstaaten auszuweichen. Erforderlich ist deshalb ein europäisches Handeln. Zwar wurde die Zusammenarbeit zwischen nationalen Aufsichtsbehörden und ESMA in den Art. 22-29 MAR geregelt. ${ }^{90}$ Die BaFin berichtet, dass sie mit 23 ausländischen Aufsichtsbehörden zusammenarbeitet. ${ }^{91}$ Gleichwohl sind Rechtshilfeersuchen zeitaufwändig, mühsam und nur selten erfolgreich. Es fragt sich auch hier, ob dies ausreichend ist und damit die erforderliche Waffengleichheit zwischen Täter und Überwacher hergestellt wird. Wenn das europäische Recht abschließend durch europäische Verordnungen geregelt werden soll, bedarf es zumindest bei grenzüberschreitenden Fällen auch entsprechender Eingriffsbefugnisse, um die notwendige Waffengleichheit gegen diese Form der organisierten Kriminalität herzustellen. In einem ersten Schritt müssten die Kompetenzen der ESMA deutlich ausgeweitet werden. Auch aus Gründen der Generalprävention wäre zu überlegen, die Zuständigkeit zu erweitern, nämlich wie die GD Wettbewerb Eingriffsmittel an die Hand zu bekommen. Ein europäisches Justizministerium wäre wahrscheinlich schlagkräftiger als eine lokale Staatsanwaltschaft. In einem zweiten Schritt könnte man auch die dritte Gewalt ausbauen, nämlich neben einem Gericht erster Instanz für Wettbewerbs-

87 Kurssturz von Wirecard am 24.2.2016; Kurssturz von Ströer am 21.4.2016; Kurssturz von Aurelius von 66 auf 45 Euro, https://boerse.ard.de/aktien/aurelius-aktie-geht-in-die-knie100. html.

88 Aktivistische Investoren sind beispielsweise Aurelius Value, Citron Research, Gotham City Research, Muddy Waters Research und Viceroy Research. Instruktiv etwa Activist Insight/Schulte Roth \& Zabel The Activist Investing Annual Review 2018, The fifth annual review of trends in shareholder activism, 2018, abrufbar unter der Webseite der Kanzlei, https://www.activistinsight. com/resources/reports/.

89 Bezieht man auch den share activism ein, kommt man auf mehr als 700 Einflussnahmen im Jahre 2016, Activist Insight/Schulte Roth \& Zabel The Activist Investing Annual Review 2017, S. 7. 90 Hierzu Thiele, Finanzaufsicht, 2014, S. 531ff.; Grundmann in Großkomm. HGB, Bankvertragsrecht, Zweiter Teil, 5. Aufl. 2017, Rn. 297 = S. 330; Zollner in Ventoruzzo/Mock, Market Abuse Regulation, 2017, B.24.01ff.

91 BaFin Jahresbericht der Bundesanstalt für Finanzdienstleistungssaufsicht 2016, 2017, S. 177. 
sachen auch ein Gericht erster Instanz für Kapitalmärkte einrichten. Drittens könnte man für die Bereiche Ad-hoc und Marktmissbrauch auch die zivilrechtliche Seite europäisch harmonisieren. ${ }^{92}$

\section{b) Zurückverlagerung von Kompetenzen an den Mitgliedstaat bei fehlendem Binnenmarktbezug}

\section{aa) Erfolgreiche Rückverlagerungen von Zuständigkeiten an die Mitgliedstaaten}

Das Prinzip der begrenzten Einzelermächtigung und das Subsidiaritätsprinzip des Art. 5 EUV wollen einem automatischen Regulierungsdrang europäischer Institutionen entgegentreten. Die Verpflichtung, Rechtsakte gem. Art. 296 Abs. 2 AEUV zu begründen, soll diese Verpflichtung zusätzlich festhalten und rationalisieren. Daneben lässt sich aber auch eine regulatorische Begründungslast aufzeigen, nämlich als Verpflichtung des Gesetzgebers, einen erforderlichen Regulierungsbedarf nachzuweisen..$^{93}$ Erfreulicherweise hat trotz der oben beschriebenen Normenflut die Europäische Union in den letzten Jahren auch harmonisierte Rechtsnormen zurückgezogen und diesen Bereich damit der Kompetenz der Mitgliedstaaten zurückgegeben. Ursprünglich sollte mit einer unionsweit einheitlichen Quartalsinformationspflicht auch eine Angleichung an die US-amerikanischen Vorschriften erfolgen, die bereits seit 1946 Quartalsberichte verlangen. ${ }^{94}$ Die Einführung dieser Quartalsberichtspflicht war allerdings auch wegen der hohen Kosten und des Einflusses saisonaler Sondereinflüsse nicht unumstritten. ${ }^{95}$ Das Erfordernis der sog. Zwischenmitteilungen, in denen das Unternehmen für das erste und das dritte Quartal des Geschäftsjahres bestimmte Finanzinformationen offen zu legen hat (Art. 6 Abs. 1 2004/109/EG a.F.), wurde durch die Transparenz-Änderungs-RiL 2013/50/EU wieder eingeschränkt. Dadurch wollte man den Verwaltungsaufwand für KMU verringern, das längerfristige Denken der Berichtspflichtigen fördern und die Informationsflut für die Anleger

92 S. einer solchen Forderung Möllers/Leisch in Hirte/Möllers, Kölner Kommentar zum WpHG, 2. Aufl. 2014, §§ 37b, c Rn. 77; Hopt WM 2012, 101, 106, 109; Wundenberg ZGR 2015, 124, $152 \mathrm{ff}$. 93 Hierzu Fleischer ZGR 2008, 185, 190, der unter andere Montesquieu zitiert: „Wenn es nicht notwendig ist, ein Gesetz zu machen, dann ist es notwendig, kein Gesetz zu machen!“.

94 Begründung des Vorschlags der EG-Kommission für eine neue Transparenz-RL v. 22.3.2003, COM (2003) 138, S. 16, 20; s. auch Merkt/Göthel RIW 2003, 23 ff.

95 Die Porsche AG hatte beispielsweise den M-DAX verlassen, s. zB SZ v. 25.3.2003, S. 20. 
zurückschrauben. ${ }^{96}$ Die privaten Regelwerke der Börsen dürfen jedoch in bestimmten Marktsegmenten weiterhin Quartalsberichte verlangen; dies bestimmt aber jeder Mitgliedstaat für sich. ${ }^{97}$ Ursprünglich verlangte die Europäische Union, alle gesellschafts- und kapitalmarktrechtlichen Veröffentlichungen wie Ad-hocMeldungen, Directors’ Dealing-Mitteilungen, Jahresabschlüsse und Zwischenberichte eines Geschäftsjahres am Ende des Jahres in einem Jährlichen Dokument aufzulisten. Mit dem am 1. Juli 2005 in Kraft getretenen Wertpapierprospektgesetz (WpPG) hatte der Gesetzgeber neue Veröffentlichungspflichten für börsennotierte Aktiengesellschaften eingeführt. Diese Informationen stehen dem Kapitalmarkt aber schon bei der jeweiligen Veröffentlichung zur Verfügung, sodass das Jährliche Dokument außer zusätzlichen Kosten keinen Mehrwert liefert. Es handelt sich um ein klassisches Beispiel einer Informationsüberflutung. ${ }^{98} \mathrm{Zu}$ Recht hat der europäische Gesetzgeber dieses wieder abgeschafft. ${ }^{99}$

\section{bb) Zulässigkeit der nationalen Besonderheiten der Anlegerberatung}

Oben wurde gezeigt, dass der deutsche Gesetzgeber durch das Vorpreschen und die Einführung zahlreicher Maßnahmen die Harmonisierung wieder torpediert; ein „level playing field“ wird damit nicht erreicht. Das Ziel, dem Verbraucher zu helfen, wird nicht erreicht, wenn die Transaktionskosten so steigen, dass die Banken auf die Anlegerberatung vollständig verzichten. M.E. hätten die Prinzipien des Art. 11 der Wertpapierdienstleistungs-RiL 93/22/EG ausgereicht, um einen europäischen Standard zu entwickeln. Daneben hat die deutsche Rechtsprechung umfangreiche Pflichten im Bereich der Bankenberatung entwickelt. ${ }^{100}$ Das eine Harmonisierung nur unzureichend möglich ist, zeigt die Gesetzgebungsgeschichte zur MiFiD II, wo die Honorarberatung aus Großbritannien und die Provisionsberatung aus Deutschland aufeinanderprallten. ${ }^{101}$ Statt das eine oder andere $\mathrm{zu}$ favorisieren, sollte jeder Mitgliedstaat für sich experimentieren, ob er den

96 Seibt/Wollenschläger ZIP 2014, 545, 546. In England und Frankreich wurde die Pflicht zur Quartalsberichterstattung bereits zuvor wieder aufgehoben, Buchheim/Hossfeld/Schmidt WPg 2016, 1347, 1350, 1352.

97 So wird etwa an der Frankfurter Wertpapierbörse gem. §53 BörsO FWB für Unternehmen im „Prime Standard“ eine sog. „Quartalsmitteilung“ verlangt. Diese ist eine verkürzte Form des vormaligen Quartalsberichts. Unternehmen im „General Standard“ sind davon befreit.

98 Möllers Juristische Methodenlehre, 2. Aufl. 2019, § 5 Rn. 148f. = S. 210.

$99 \S 10$ WpHG wurde gestrichen durch das Gesetz zur Umsetzung der Richtlinie 2010/73/EU und zur Änderung des Börsengesetzes v. 26.06.2012, BGBl. I S. 1375.

100 Hierzu etwa Möllers/Leisch in Gerke/Steiner, Handwörterbuch des Bank- und Finanzwesens, 3. Aufl. 2001, S. $311 \mathrm{ff}$.

101 Hierzu ausführlich Möllers ZEuP 2016, 325, 341 ff. 
Bankkunden mit einem Beratungsprotokoll, einem Produktinformationsblatt, umfangreichen Vorgaben an Provisionserstattung (kick-back) oder einem Beschwerderegister beglücken möchte. Binnenmarktrelevant sind solche Vorgaben nicht.

\section{Die Umsetzung europäischer Rechtsprinzipien und kapitalmarktrechtlicher Regelungsziele}

\section{a) Subsidiaritätsprinzip des Art. 5 Abs. 3 EUV}

Mit einer solchen horizontalen, binnenmarktrelevanten Arbeitsteilung würde auch das Subsidiaritätsprinzip nach Art. 5 Abs. 3 EUV inhaltlich ernst genommen: Bei der Marktmanipulation liegen die Vorgaben des Subsidiaritätstests zugunsten der Europäischen Union vor: Der Subsidiaritätstest negativ geprüft besagt, dass der Mitgliedstaat das Problem nur unzureichend lösen kann und deshalb die Europäische Union als effektivere Einheit gefordert ist, nämlich bei grenzüberschreitenden Markmanipulationen vorzugehen. Letztlich wird Art. 12 MAR in gleicher Weise wie der frühere $\S 20$ a WpHG und der frühere $\S 88$ BörsG bei grenzüberschreitenden Sachverhalten gegenüber irreführenden Research Reports häufig wirkungslos bleiben. Die Wirkungslosigkeit des Kapitalmarktrechts beschädigt aber das Vertrauen in die Kapitalmärkte. Deshalb ist eine europäische Rechtsdurchsetzung vonnöten.

Aber auch umgekehrt gilt: Wenn das berühmte „Lieschen Müller von der Raiffeisenbank Buxtehude“ eine Anleihe in Höhe von 1.000 EUR erwirbt, ist dies weder systemrelevant noch muss hierfür wirklich eine Verordnung oder eine europäische Richtlinie herhalten. Das Subsidiaritätsprinzip verlangt aber positiv, die Kompetenz auf der tieferen Ebene zu belassen, wenn diese die Problematik besser lösen kann. Hier drängt sich geradezu der Vergleich mit der Keck-Rechtsprechung des EuGH auf. Ob englische Pubs nach 22.00 Uhr noch Bier ausschenken oder Deutschland am Sonntag die Geschäfte geschlossen hält, sind vertriebsbezogene Maßnahmen, die den Binnenmarkt nicht tangieren und insoweit aus dem Schutzbereich der Warenverkehrsfreiheit herausfallen. ${ }^{102}$ Deshalb sollte im Bereich der Anlageberatung jedes Mitglied selbst regeln, unter welchen Voraussetzungen eine solche zulässig ist.

102 S. oben Fn. 75 und W. Schroeder in Streinz, EUV/AEUV, 3. Aufl. 2018, Art. 34 AEUV Rn. 41 ff., 45; Kingreen in Callies/Ruffert, EUV/AUEV, 5. Aufl. 2016, Art. 34-36 AEUV Rn. 49 ff. 


\section{b) Die doppelte Effizienzsteigerung}

Die positive wie negative Seite des Subsidiaritätsprinzips wäre damit auch in doppelter Weise effizienzsteigernd: Ein europäisches Einschreiten würde dort helfen, wo nationale Behörden überfordert sind (III.b)i)). Umgekehrt würden Ressourcen und Transaktionskosten gespart, wenn nationales oder lokales Handeln ausreicht (III.b)ii)). Der europäische Gesetzgeber würde so die Wettbewerbsfähigkeit von Unternehmen und Banken gegenüber ihren US-amerikanischen und chinesischen Wettbewerbern nicht schwächen, sondern durch eine klug verstandene Deregulierung stärken. ${ }^{103}$ Zugleich würde in Zeiten des Brexits aufgezeigt, wie ein zukunftsfähiges Europa aussehen könnte.

\section{Zusammenfassung}

1. Das europäische und nationale Kapitalmarktrecht ist schon auf der Gesetzgebungsebene schnelllebig und verdichtet sich mit dem Lamfalussy- und LarosièreVerfahren zu sechs rechtlich relevanten Ebenen. Das sind die Rahmenakte, Durchführungsakte und das Recht der ESMA auf europäischer Ebene. Hinzu kommen auf nationaler Ebene die Gesetze, Verordnungen und dann das Verwaltungsinnenrecht wie etwa der Emittentenleitfaden. Diese doppelte Komplexität wird erweitert um vier Besonderheiten, die sich aus dem Verhältnis von europäischem zum nationalen Recht ergeben: der Problematik von Mindest- und Vollharmonisierung, dem Vorpreschen des nationalen Gesetzgebers, dem Verhältnis von Richtlinien und unmittelbar geltenden Verordnungen und dem Einfluss des europäischen Rechts auf das nicht harmonisierte Recht. Die Praxis zeigt sich überfordert und wartet auf die neue Fassung des Emittentenleitfadens oder des FSA Handbook.

2. Diese Komplexität geht einher mit einer Komplexität der juristischen Methoden bei der Rechtsanwendung. So wird man streng zwischen der verordnungskonformen und richtlinienkonformen Auslegung unterscheiden müssen. Auch die Frage des Vorrangs ist abhängig von der Hierarchie des europäischen Gesetzes. Umgekehrt macht es komplexes und schnelllebiges europäisches Recht unmöglich, eine Rechtsdogmatik zu entwickeln, die zur Lückenfüllung unabdingbar ist.

3. De lege ferenda ist im Kapitalmarktrecht eine Arbeitsteilung zwischen den Mitgliedstaaten und der Europäischen Union herzustellen. Man könnte durch

$103 \mathrm{Zu}$ dieser Zielsetzung s. bereits oben I.c)ii). 
eine Stärkung sowohl der europäischen als auch nationalen Ebene eine Win-winSituation schaffen. Durch die Trennung der Zuständigkeiten könnte die Verantwortlichkeit erhöht werden, aber auch die jeweils eigene Rechtsetzung und Rechtsdurchsetzung gestärkt werden. Auf europäischer Ebene müsste die Rechtsdurchsetzung für die Bereiche von Insiderrecht und Marktmissbrauchskontrolle weiter verbessert werden. Auf nationaler Ebene könnte ein Wettbewerb der Mitgliedstaaten in Bereichen eintreten, in denen der Binnenmarkt und systemische Risiken nicht berührt sind, wie etwa im Bereich der Anlegerberatung. Damit würden auf europäischer Ebene systemrelevante Bereiche effektiver gemanagt, auf nationaler Ebene würde das Recht einfacher, weil die vier Ebenen des europäischen Rechts wegfielen.

4. Die jetzige Fassung des WpHG ist sprachlich schon wenig überzeugend ${ }^{104}$; als „entleertes Verweisungsgesetz“ spielt die Musik künftig bei den unmittelbar geltenden europäischen Verordnungen. Wenn der Rechtsanwender auf den Emittentenleitfaden als verständige Rechtsquelle wartet, so sollte es auch für den deutschen Gesetzgeber ein Ansporn sein, verständliches Recht zu schaffen. Würde man umfangreiche Bereiche der MiFiD II wieder vollständig der Kompetenz der Mitgliedstaaten überlassen, hätte das Wertpapierhandelsgesetz eine realistische Chance die 50 Jahresfeier zu erleben - als Quelle nationalen Rechts, konkretisiert durch die BaFin und den Bankensenat des BGH.

104 S. etwa Art. 6 Abs. 5, § 10, § 25, § 53 oder auch die $\S \S 119$ f. WpHG. 